\title{
Lealdade à Marca e Sensibilidade ao Preço: um Estudo da Escolha da Marca pelo Consumidor
}

\author{
Delane Botelho \\ André Torres Urdan
}

\section{Resumo}

Este artigo empírico usa dados escaneados em painel de domicílios para estimar e analisar a elasticidade-preço da escolha da marca em dois grupos de clientes: os leais e os não leais à marca de uma categoria de bem de consumo. O objetivo principal é verificar diferenças de sensibilidade ao preço entre os grupos de consumidores na escolha da marca. O método usado para a análise dos dados é o da Máxima Verossimilhança, com a abordagem da maximização da utilidade, empregandose o modelo logit misto, com dois tipos de vetores de parâmetros: o de características dos domicílios e o de atributos das marcas. Os resultados indicam que a diferença de sensibilidade ao preço entre os consumidores varia de acordo com as marcas. Com isto, conclui-se que profissionais de marketing podem tomar decisões mais efetivas de apreçamento levando em conta as características dos domicílios e os atributos das marcas. Considerando que a disponibilidade de dados escaneados no Brasil aumentou consideravelmente desde o início da década de 1990, esta ferramenta de análise pode ser útil ao setor varejista brasileiro.

Palavras-chave: elasticidade-preço; modelo logit; dados escaneados; escolha do consumidor.

\begin{abstract}
This empirical paper applies scanner panel data to estimate brand choice price-elasticities for two groups of households: loyal and non-loyal consumers to a brand of a consumer good. The main objective is to verify differences in price sensitiveness among those consumers when they choose brands. The method of Maximum Likelihood and the approach of utility maximization are used to estimate the mixed logit model with two types of parameter vectors: household characteristics and brand attributes. The results show that differences in price elasticities among consumers vary according to each brand, implying that marketing managers can take more effective decisions when considering household characteristics and brand attributes. Such analysis can be helpful for the Brazilian retail sector, as the availability of scanner data has been increasing since last decade.
\end{abstract}

Key words: price-elasticity; logit model; scanner data; consumer choice. 


\section{INTRODUÇÃO}

As relações entre as variáveis mercadológicas e o desempenho de marketing são de considerável interesse para os profissionais dessa disciplina e dos pesquisadores dessa ciência. Ambos estão interessados na resposta do mercado às variáveis do composto de marketing: preço, produto, praça e promoção. Querem saber como seus esforços influenciam a escolha do consumidor pelos seus produtos e, conseqüentemente, as medidas de desempenho da empresa (como as vendas, o lucro e a participação de mercado), já que estas têm impacto direto na sobrevivência das empresas. A escolha do consumidor é área predominante de pesquisa em marketing (CHINTAGUNTA et al., 2001) e este artigo $^{(1)}$ trata da escolha do domicílio influenciada por uma das variáveis do composto de marketing: o preço. O principal objetivo do artigo é verificar se domicílios leais e não leais à marca de um bem de consumo geral são diferentemente sensíveis às promoções de preços de suas marcas preferidas e das demais para a escolha da marca. Outros objetivos secundários são: 1) verificar se a elasticidade-preço da escolha da marca varia entre as marcas analisadas; 2) inferir a relação entre renda domiciliar e preços das marcas escolhidas pelos domicílios; 3) oferecer base metodológica para a estimativa da elasticidade-preço da escolha da marca, usando dados escaneados, sob uma perspectiva econométrica aplicada ao marketing.

A hipótese principal a ser testada é a de que compradores leais a uma marca tendem a ser insensíveis à variação de preço da referida marca na escolha da marca a comprar. Esta é, segundo Krishnamurti e Raj (1991), uma premissa simples e fácil de ser aceita, pois existe uma relação estreita entre a lealdade à marca e a sensibilidade ao preço. Esta relação é válida apenas para um componente do processo de decisão: a escolha da marca (e não a quantidade a comprar). Portanto a hipótese H1 é:

H1. Domicílios leais a uma marca são menos sensíveis à variação de preços na escolha da marca do que os domicílios não leais.

A segunda hipótese a ser testada envolve a relação entre renda do domicílio e escolha da marca influenciada pelo preço. Renda é uma variável demográfica, que capta parte da heterogeneidade da amostra e tende a ser negativamente relacionada à intensidade de pesquisa de preços, pois compradores com alta renda, geralmente, apresentam alto custo de oportunidade do tempo (BLATTBERG et al., 1978). Supondo um mercado competitivo em que os 
produtores tenham acesso às mesmas tecnologias de produção, Allemby e Rossi (1991) afirmam que há alta correlação entre preço e qualidade e que os consumidores maximizam a utilidade também buscando qualidade, conforme a restrição orçamentária. Daí surge a hipótese H2:

H2. Domicílios com rendas mais altas apresentam maior probabilidade de escolha de marcas de preço mais alto.

Este artigo pretende ser original no Brasil na utilização de dados escaneados em modelos de estimação da elasticidade-preço da escolha da marca, que incluem o construto lealdade à marca e variáveis demográficas, como a renda. Seu tema central envolve a escolha do consumidor pela marca, tendo o preço como principal variável mercadológica influenciadora desta resposta.

O artigo está assim estruturado: a primeira seção trata da revisão de literatura sobre apreçamento, com foco na elasticidade-preço e no modelo logit para seu cálculo; a segunda seção aborda o método de pesquisa, com a coleta de dados, as variáveis utilizadas e a especificação do modelo proposto. Os resultados são discutidos na terceira seção e o artigo é finalizado com as conclusões, implicações gerenciais e sugestões para pesquisas futuras.

\section{REVISÃo dA LiteratuRA}

O preço tem sido estudado sob várias perspectivas e níveis de intensidade, principalmente pela economia, finanças, marketing e mesmo psicologia. Parece, entretanto, que, apesar de o marketing satisfazer melhor o critério pragmatismo em relação à economia no estudo do preço, a ciência do marketing tem contribuído muito pouco para a eficácia de apreçamento das empresas. Simon (1989, p. 40) relata grande discrepância entre a teoria e a prática no estudo do preço, devido principalmente à negligência aos problemas de informação, quantificação e implementação de modelos teóricos de precificação. No Brasil, Rocha e Christensen (1999, p. 111) mencionam que a determinação de preços pelo custo total é provavelmente o método mais utilizado pelas empresas na fixação do preço básico de seu produto, em detrimento de abordagem mais abrangente, incluindo o papel do consumidor e suas percepções.

As decisões de preços nas últimas décadas no Brasil sofreram o impacto de fatores ambientais, que moldaram a forma pela qual elas foram tomadas. Rocha e Christensen (1999, p. 107) destacam a inflação acelerada das décadas de 1970, 1980 e início de 1990 e, mais recentemente, o processo de 
desregulamentação da economia. A Tabela 1 apresenta uma série histórica do Índice de Preços ao Consumidor da Fundação Instituto de Pesquisas Econômicas, conveniada com a Universidade de São Paulo. Somente a partir de 1997 este índice de inflação ficou abaixo dos 10\% ao ano. Até o início da década de 1990, além das elevadas taxas de inflação, empresas e compradores conviviam com mudanças abruptas nestas taxas, devido aos planos governamentais para o controle da inflação, o que contribuiu para desorganizar ainda mais os preços relativos de bens e serviços. Em decorrência, o estudo acadêmico do preço, como elemento do composto mercadológico, perdeu importância no Brasil naquela época. Já na década de 1990, a queda da inflação veio revitalizar o preço e desafiar as empresas brasileiras a melhorarem suas práticas de apreçamento (ROCHA; CHRISTENSEN, 1999, p. 108).

Tabela 1: Evolução do Índice de Preços ao Consumidor (1993-2004)

\begin{tabular}{rr}
\hline Ano & Inflação \\
\hline 1993 & $2.490,5$ \\
1994 & 941,0 \\
1995 & 23,0 \\
1996 & 10,6 \\
1997 & 4,8 \\
1998 & $-1,8$ \\
1999 & 8,6 \\
2000 & 4,4 \\
2001 & 7,1 \\
2002 & 9,9 \\
2003 & 8,2 \\
2004 & 6,6 \\
\hline
\end{tabular}

Fonte: Fundação Instituto de Pesquisas Econômicas (2004).

O uso de dados escaneados, obtidos por meio de leitura ótica dos códigos de barras dos produtos e cartões de fidelidade dos clientes, também ganhou espaço no varejo brasileiro na década de 1990, facilitando o estudo acadêmico de preço. O número de lojas de supermercados com caixas dotados de leitura ótica atingiu 17,6 mil em 1999 (de um total de 24 mil lojas em todo o Brasil), um aumento de 26\% em relação ao ano anterior. O número de produtos identificados com código de barras também cresceu, saltando de 410 mil para 500 mil, uma expansão de 22\% de 1998 para 1999 (ARBEX, 2000). Também cresceu o número de lojas providas de scanners de código de barras nas 500 maiores redes supermercadistas brasileiras, passando de 15 lojas em 1991 para 3.174 no final de 2001, como 
mostra o Gráfico 1. Tal crescimento, entretanto, não foi acompanhado pela produção de conhecimento científico, usando essa poderosa ferramenta no Brasil.

\section{Gráfico 1: Evolução do Número de Lojas Providas de Scanners nas 500 Maiores Redes Supermercadistas Brasileiras}

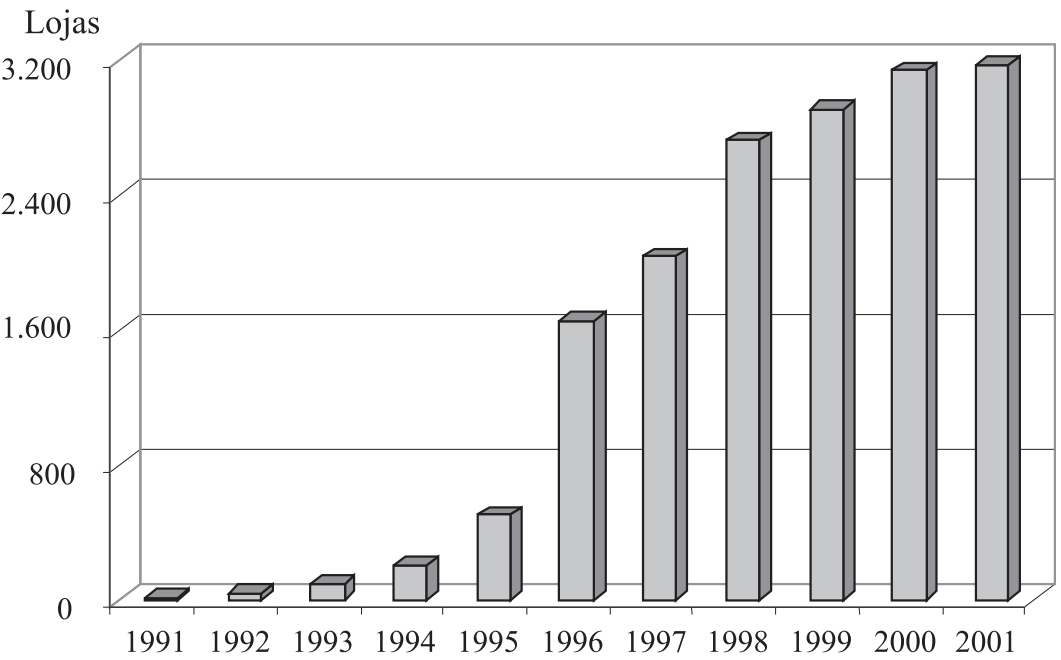

Fonte: Associação Brasileira de Supermercados (2002).

Ano

Na literatura internacional de marketing, dados escaneados têm sido usados intensivamente na estimação da elasticidade-preço da demanda, um dos tópicos mais importantes do preço em marketing, como nos trabalhos de Guadagni e Little (1983), Gupta (1988), Tellis (1988), Krishnamurthi e Raj (1991), Bell e Lattin (1998) e Chintagunta et al. (2001). Por isto mesmo tal conceito é a seguir examinado.

\section{O Conceito de Elasticidade-preço}

A medida mais utilizada e aceita na avaliação da sensibilidade do consumidor ao preço é a elasticidade-preço da demanda (EPD), que representa a alteração percentual na quantidade vendida, dada uma alteração percentual no preço:

$E P D=\frac{\Delta \% Q}{\Delta \% P}=\frac{\text { variação percentual na quantidade demandada }}{\text { variação percentual no preço }}$

Dois modos distintos de cálculo são utilizados para a determinação do valor da EPD: a elasticidade no arco e a elasticidade no ponto (LIPSEY et al., 1999, p. 
332). A primeira é uma medida aproximada da segunda, pois mede, num dado intervalo da curva de demanda, a sensibilidade média da quantidade demandada em face da variação do preço. Deste modo, $\mathrm{P}$ e Q na equação 1 são representados por $\overline{\mathrm{P}}$ e $\overline{\mathrm{Q}}$, respectivamente. A elasticidade no ponto mede, num ponto específico da curva de demanda, a sensibilidade da quantidade demandada em face da variação do preço. Esta é determinada pelo cálculo diferencial e a sua expressão algébrica é a seguinte:

$E P D=\frac{\partial \mathrm{Q} / \mathrm{Q}}{\partial \mathrm{P} / \mathrm{P}}=\frac{\partial \mathrm{Q} / \partial \mathrm{P}}{\mathrm{Q} / \mathrm{P}}$

em que $\mathrm{P}$ e Q constituem o preço e a quantidade no ponto de interesse na curva de demanda e $\partial \mathrm{Q} / \partial \mathrm{P}$ representa a derivada da quantidade em relação ao preço neste mesmo ponto. A derivada exprime a variação da quantidade resultante da variação unitária do preço. O valor da elasticidade no ponto é único, porque existe apenas uma única tangente à curva de demanda no ponto referenciado. A teoria microeconômica em geral utiliza-se apenas da elasticidade no ponto, que é a aplicada neste artigo. Para um intervalo pequeno da curva de demanda, a equação 2 pode ser usada como elasticidade no ponto sem grandes margens de erro.

A abordagem de elasticidade apresentada até aqui refere-se à forma mais comum encontrada na literatura econômica e de marketing. Entretanto a decisão de compra de uma marca pelo consumidor não se dá apenas na dimensão da quantidade a ser comprada, mas também na da marca a comprar (KRISHNAMURTHI; RAJ, 1988). O foco deste artigo é a elasticidade-preço da escolha da marca (EPEM), que mede o efeito do preço não sobre a quantidade comprada, mas sobre a probabilidade de se escolher determinada marca. Esta elasticidade será explicada a seguir.

\section{O Modelo Logit e a Elasticidade-Preço da Escolha da Marca (EPEM)}

Qualquer escolha do comprador é, por definição, feita a partir de um conjunto não vazio de alternativas. A atratividade de uma alternativa é avaliada pelo indivíduo em termos de um vetor de valores de atributos. No caso deste artigo, os indivíduos (domicílios) podem avaliar diferentes atributos para as alternativas (marcas) e atribuir diversos valores para o mesmo atributo da mesma alternativa.

Aqui aplica-se o conceito de utilidade (U) ao modelo de escolha do domicílio. Manski (1977) (apud BEN-AKIVA; LERMAN, 1985, p. 238) formalizou a abordagem de utilidade aleatória, que melhor reflete a realidade dos estudos 
empíricos. Considerando-se apenas duas alternativas (modelo binário) e sabendose que $U_{\text {in }}$ e $U_{i j}$ são variáveis aleatórias, tem-se:

$\mathbf{U}_{i n}=V_{i n}+\varepsilon_{i n}$

$\mathbf{U}_{j n}=\mathbf{V}_{j n}+\varepsilon_{j n}$

$\mathrm{V}_{\text {in }}$ e $\mathrm{V}_{j n}$ são funções e referem-se aos componentes determinísticos da utilidade das alternativas $i$ e $j$, ou seja, podem ser tratados como as médias de $\mathrm{U}_{\text {in }}$ e $\mathrm{U}_{\mathrm{ij}}$. $\varepsilon_{\text {in }}$ e $\varepsilon_{\text {in }}$ também podem ser funções e referem-se aos componentes aleatórios das utilidades de $i$ e $j$.

Na especificação dos componentes determinísticos da utilidade $\left(V_{i n}\right)$, para o indivíduo $n$ qualquer alternativa $i$ pode ser caracterizada por um vetor de atributos $\mathbf{z}_{\text {in; }}$; no caso de uma marca, ele pode ser composto das variáveis preço e qualidade, por exemplo. É também útil caracterizar o indivíduo $n$ por outro vetor de atributos, $\mathbf{s}_{\mathrm{n}}$, formado por renda, idade e ocupação, por exemplo. Sendo $\mathbf{x}$ o vetor que inclui $\mathbf{z}_{\text {in }}$ e $\mathbf{s}_{\mathrm{n}}$, a parte determinística da utilidade pode ser definida como $V_{i n}=\beta^{\prime} x_{i n}$, para o indivíduo $n$ e a alternativa $i$, onde $\beta$ se refere ao vetor de parâmetros a ser estimado. Neste caso $V_{\text {in }}$ é uma função linear dos parâmetros.

O modelo logit é usado quando se pressupõe que $\varepsilon_{n}=\varepsilon_{i n}-\varepsilon_{j n}$ (nas equações 3 e 4) possui distribuição logística:

$\Phi\left(\varepsilon_{n}\right)=F\left(\varepsilon_{n}\right)=\frac{1}{1+e^{-\mu \varepsilon_{n}}}, \mu>0,-\infty<\varepsilon_{n}<\infty$, (Função de Distribuição Acumulada) (5)

$\phi\left(\varepsilon_{n}\right)=f\left(\varepsilon_{n}\right)=\frac{\mu e^{-\mu \varepsilon_{n}}}{\left(1+e^{-\mu \varepsilon_{n}}\right)^{2}}, \quad \quad$ (Função Densidade de Probabilidade) (6)

onde $\mu$ é um parâmetro positivo. Além de ser uma aproximação da distribuição normal, a distribuição logística é analiticamente conveniente, se comparada ao modelo probit, que envolve múltiplas integrais.

Assumir que $\varepsilon_{n}$ possui distribuição logística é equivalente a assumir que $\varepsilon_{\text {in }}$ e $\varepsilon_{j n}$ são independentes e identicamente distribuídos pela distribuição Gumbel (ou tipo I de valores extremos). Se $\varepsilon$ possui distribuição Gumbel, então: $F(\varepsilon)=\exp \left[-e^{-\mu(\varepsilon-\eta)}\right], \mu>0$ e $f(\varepsilon)=\mu e^{-\mu(\varepsilon-\eta)} \exp \left[-e^{-\mu(\varepsilon-\eta)}\right]$, onde $\eta$ refere-se ao parâmetro de locação e $\mu$ ao parâmetro de escala.

Sob essa pressuposição, a probabilidade de escolha da alternativa $i$ em relação à alternativa $j$ (modelo binário) é dada por:

$\mathrm{P}_{n}(i) \quad=\operatorname{Pr}\left(\mathrm{U}_{i n} \geq \mathrm{U}_{j n}\right) \quad=\frac{1}{1+e^{-\mu\left(V_{i n}-V_{j n}\right)}}=\frac{e^{\mu V_{i n}}}{e^{\mu V_{i n}}+e^{\mu V_{j n}}}=\frac{e^{\mu \beta^{\prime} x_{i n}}}{e^{\mu \beta^{\prime} x_{i n}}+e^{\mu \beta^{\prime} x_{j n}}}$ 
No caso de utilidades lineares nos parâmetros $\beta$, o parâmetro $\mu$ não se distingue de $\beta$. Logo, por conveniência, assume-se arbitrariamente a igualdade $\mu=1$ (BEN-AKIVA; LERMAN, 1985, p. 256).

A teoria vista até aqui se restringe aos casos em que o conjunto de escolha possui apenas duas alternativas. Entretanto, neste trabalho empírico, o consumidor defronta-se com um conjunto de quatro marcas $(\mathrm{J}=4)$. Um modelo multinomial misto, que é o usado neste artigo, apresenta dois tipos de vetores de parâmetros, sendo um de características individuais e o outro de atributos das alternativas:

$P_{n}(i)=\frac{e^{V_{i n}}}{\sum_{j \in C_{n}} e^{V_{j n}}}=\frac{e^{\beta x_{i n}}}{\sum_{j \in C_{n}} e^{\beta^{\prime} x_{j n}}}$

Tal modelo se reduz ao modelo logit binário quando $\mathrm{J}=2$. $\beta$ está representando os dois vetores: características e atributos. A elasticidade-preço da escolha da marca (EPEM) pode ser obtida por meio do modelo logit por:

$E P E M=\frac{\partial P_{n}(i)}{\partial x_{i n k}} \times \frac{x_{i n k}}{P_{n}(i)}=\frac{\partial \ln P_{n}(i)}{\partial \ln x_{i n k}}=\left[1-P_{n}(i)\right] x_{i n k} \beta_{k}$

De modo similar, elasticidade cruzada no modelo logit é dada por:

$\operatorname{EPEM}_{C}=\frac{\partial \ln P_{n}(i)}{\partial \ln x_{j n k}}=\left[1-P_{n}(i)\right] x_{j n k} \beta_{k}$, para $j \neq i$

onde $n$ se refere ao indivíduo, $i$ se refere à alternativa e $k$ ao subscrito da variável de interesse. $\mathrm{P}_{n}(i)$ é a probabilidade do indivíduo $n$ escolher a alternativa $i$.

\section{MÉTodo}

Este artigo utiliza dados escaneados em painel, definidos por Johnston e Dinardo (1997, p. 388) como compostos de observações no mesmo conjunto de seções cruzadas, mas que se repetem ao longo do tempo. Sendo $\mathrm{N}$ o número de indivíduos e T o número de ocasiões de compra, quando:

$\mathrm{N}=1$ e $\mathrm{T}>1$, tem-se as séries temporais;

$\mathrm{N}>1$ e $\mathrm{T}=1$, tem-se as seções cruzadas;

$\mathrm{N}>1$ e $\mathrm{T}>1$, tem-se dados em painel, sendo $\mathrm{N}>\mathrm{T}$. 
Os dados aqui correspondem a um painel desbalanceado, isto é, com números diferentes de situações de compra para cada domicílio. Esta característica devese à exclusão do banco de dados daquelas ocasiões em que não se observou compra, pelo domicílio, nas marcas estudadas dentro da categoria de produto analisada.

Foi usada uma combinação das ocasiões de compra dos diversos domicílios, como numa seção cruzada, em que cada ocasião de compra representou uma observação independente. Trata-se, portanto, de uma combinação de seção cruzada com série temporal, seguindo o método dos artigos que usam dados do tipo painel e modelos probabilísticos com mais de duas alternativas (GUADAGNI; LITTLE, 1983; KRISHNAMURTHI; RAJ, 1988, 1991). Portanto, considera-se cada visita do cliente à loja (que resulte em compra na categoria) como uma observação independente.

\section{Coleta de Dados}

Os dados foram coletados numa rede supermercadista da região sudeste do Brasil para quatro marcas (A, B, C e D) da categoria de achocolatados em pó. Os dados são desagregados no nível de domicílio, obtidos por meio do cartão de fidelidade utilizado pelo varejista. As quatro marcas são responsáveis por mais de $80 \%$ das vendas nesta categoria. A forma dominante de promoção na categoria é a redução de preços. A Tabela 2 apresenta a participação de mercado de cada marca dentro da rede varejista e seus preços médio, máximo e mínimo durante o período analisado.

Tabela 2: Participação de Mercado de Cada Marca na Rede Varejista e Seus Preços Médio, Máximo e Mínimo durante o Período de Análise

\begin{tabular}{ccccc}
\hline Marca & Participação \% & Preço Médio & Preço Máximo & Preço Mínimo \\
\hline A & 81,7 & 2,17 & 2,90 & 1,64 \\
B & 9,7 & 2,28 & 2,49 & 2,08 \\
C & 6,8 & 1,14 & 1,45 & 0,85 \\
D & 1,8 & 1,62 & 2,05 & 1,39 \\
\hline
\end{tabular}

Uma amostra de 4.000 clientes foi obtida para análise inicial, durante um período de 35 semanas. Somente foram considerados os clientes que realizaram mais de 10 compras nas marcas analisadas no período. Este método está coerente com a literatura de marketing. Segundo Ainslie e Rossi (1998), o número médio de observações por domicílio em um estudo típico de dados escaneados é menor que 20, com grande parte de domicílios com menos de dez observações. Excluindose os clientes com missing values, restou uma amostra aleatória de 200 clientes em 2.604 situações de compra na categoria. Portanto a média de visitas às lojas que resultaram em compra na categoria foi de 13,02. 


\section{Variáveis Utilizadas}

As variáveis utilizadas no modelo estão a seguir apontadas e definidas.

Lealdade $\left.(\mathbf{L E A L})_{p}\right)$ - há várias definições teóricas do construto lealdade para uso em modelos quantitativos de marketing. Uma delas, muito usada, tem a lealdade como medida de preferência pela marca (GUADAGNI; LITTLE, 1983; GUPTA, 1988). Tais autores captaram o comportamento leal à marca via uma média exponencialmente ponderada de todas as compras anteriores à ocasião $t$. Já Krishnamurti e Raj (1991) captaram o comportamento leal como a proporção de compras da marca anterior à ocasião $t$. Bucklin e Gupta (1992) utilizaram simplesmente a última marca comprada para captar a lealdade. Neste estudo, optou-se pelo método de Krishnamurti e Raj (1991), que usa uma medida comportamental (e não atitudinal) da lealdade, baseada na proporção de compras do domicílio dedicada a cada marca. Primeiro foi operacionalizada a variável $\operatorname{PROP}_{p}$ como a proporção de vezes que cada marca foi comprada por cada domicílio, em relação às compras anteriores, nas oito primeiras semanas do período de 35 semanas. Por exemplo, se o valor de $\mathrm{PROP}_{p}$ fosse 0,8 , então o domicílio seria $80 \%$ leal à marca $p$, ou seja, de cada cinco compras na categoria, quatro teriam sido da marca $p$. O ideal seria que o período de estabilização da variável $\mathrm{PROP}_{p}$ não coincidisse com o período de calibração do modelo, mas a escassez de dados ao longo do tempo impôs esta limitação. Krishnamurthi e Raj (1991) e Tellis (1988), como dispunham de longa série temporal, usaram 20 semanas anteriores ao período de calibração como período de estabilização desta variável. Por outro lado, Chiang (1991) dispunha de apenas 24 semanas e usou as quatro primeiras para estabilizar a variável lealdade. Então, a variável categórica LEAL foi criada da seguinte maneira:

$$
\begin{array}{lll}
\operatorname{LEAL}_{p}=1 & \text { se } & \operatorname{PROP}_{p}>0,7 \\
\operatorname{LEAL}_{p}=0 & \text { se } & \operatorname{PROP}_{p}<0,3 .
\end{array}
$$

Os domicílios para os quais $\mathrm{PROP}_{p}$ apresentou valores entre 0,3 e 0,7 foram excluídos da amostra final, já que uma característica do banco de dados era o grande número de observações em seção cruzada, o que possibilitou a exclusão de muitos domicílios, apesar da escassez de informações em série temporal. Assim incluíram-se na amostra apenas os domicílios claramente leais ou não leais, compensando a limitação da falta de um período longo e definido de estabilização deste vetor de variáveis.

Preço por 100 gramas $\left(\mathbf{P R E C ̧ O}_{p}\right)$ - primeiro foi obtido o preço por item da marca. Quando o domicílio comprava determinada marca, o preço da marca era o preço pago pelo domicílio. Os preços das outras três marcas entraram no banco de dados naquela ocasião de compra para que as elasticidades-preço 
cruzadas fossem calculadas. Estes preços eram os vigentes no dia em que o domicílio realizou a compra na loja específica. A variável PREÇO ${ }_{p}$ foi calculada dividindo-se o preço da embalagem da marca comprada pelo peso da embalagem em gramas e multiplicando-se o resultado por 100. Desta maneira trabalhou-se com uma variável contínua (preço por 100g).

Interação $\mathbf{L E A L}_{P} * \mathbf{P R E C ̧ O}_{P}$ : como o principal objetivo deste artigo é verificar as diferenças de lealdade à marca na sensibilidade ao preço, introduziu-se uma variável para captar a interação da lealdade com o preço. Segundo Gujarati (2000, p. 516), para estimar duas equações de regressão, uma para os clientes leais à marca (variável $\operatorname{LEAL}_{p} \geq 0,7$ ) e outra para aqueles não leais, tem-se:

Clientes não leais:

$$
y_{i}=\gamma_{1}+\gamma_{2} x_{i}+u_{1 i} \quad i=1,2, \ldots, n_{1}
$$

Clientes leais:

$$
y_{i}=\lambda_{1}+\lambda_{2} x_{i}+u_{1 i} \quad i=1,2, \ldots, n_{2}
$$

sendo $y$ as vendas e $x$ o preço. Unindo-se todas as $n_{1}$ e $n_{2}$ observações, pode-se estimar a seguinte regressão:

$$
Y_{i}=\alpha_{1}+\alpha_{2} D_{i}+\beta_{1} x_{i}+\beta_{2}\left(D_{i} x_{i}\right)+u_{i}
$$

onde $\mathrm{D}_{\mathrm{i}}=1$ para observações cujos clientes são leais à marca e $\mathrm{D}_{\mathrm{i}}=0$ para aqueles não leais. Para entender as implicações da equação 13, e admitindo-se que $\mathrm{E}\left(u_{i}\right)=0$, tem-se:

Clientes não leais: $\quad E\left\langle y_{i} \mid D_{i}=0, x_{i}\right\rangle=\alpha_{1}+\beta_{1} x_{i}$

Clientes leais:

$$
E\left\langle y_{i} \mid D_{i}=1, x_{i}\right\rangle=\left(\alpha_{1}+\alpha_{2}\right)+\left(\beta_{1}+\beta_{2}\right) x_{i}
$$

que são, respectivamente, as funções de demanda para os clientes não leais e para os leais. As equações 14 e 15 são absolutamente iguais às equações 11 e 12, com $\gamma_{1=} \alpha_{1,} \gamma_{2=} \beta_{1,} \lambda_{1=}\left(\alpha_{1}+\alpha_{2}\right)$ e $\lambda_{1=}\left(\beta_{1}+\beta_{2}\right)$. Logo, estimar a equação 13 equivale a estimar as funções demandas individuais (equações 14 e 15).

Na equação 13, $\alpha_{1}$ é o coeficiente do intercepto diferencial e $\beta_{2}$ é o coeficiente da inclinação diferencial, indicando em quanto o coeficiente de inclinação da função demanda para os clientes leais difere do coeficiente da função demanda para os clientes não leais (GUJARATI, 2000, p. 516).

A introdução da variável dummy D na forma multiplicativa (D multiplicado por $x$ ) permite diferenciar os coeficientes de inclinação dos dois grupos de clientes, 
assim como a introdução da variável dummy na forma aditiva permite distinguir os interceptos dos dois períodos. Se $\beta_{2}$ for estatisticamente significativo, o coeficiente de inclinação do grupo de clientes leais será $\beta_{1}=\beta_{2}$, sendo $\beta_{1}$ o coeficiente de inclinação do grupo dos não leais. Se $\beta_{2}$ for estatisticamente não significativo, então $\beta_{1}$ representa a inclinação para os dois grupos de clientes.

Renda (característica demográfica): a relação da elasticidade-preço com algumas variáveis demográficas ainda gera controvérsia na literatura. Bucklin e Gupta (1992) evidenciam fraca relação entre as variáveis demográficas dos domicílios e a sensibilidade às variáveis do composto mercadológico, quando dados escaneados são utilizados. Já Ainslie e Rossi (1998) concluíram que características demográficas dos consumidores têm alta correlação com a EPD. Neste artigo, a variável demográfica utilizada foi a renda per capita, obtida dividindo-se a renda familiar pelo número de componentes da família.

\section{Especificação do Modelo}

As funções de utilidade para as quatro marcas são dadas por:

$U_{P}=\beta_{0 P}+\beta_{P} \operatorname{Preço~}_{P}+\beta_{4+P}$ Renda $+\beta_{8+P} L E A L_{P}+\beta_{12+P}\left(L E A L_{p} * \operatorname{Preço}_{P}\right)$

tendo-se $p=1,2,3,4$, para as marcas A, B, C e D, respectivamente.

Modelos probabilísticos trazem dificuldades especiais para a avaliação da adequação geral do modelo. Enquanto os modelos de regressão linear apresentam os resíduos e o $R^{2}$ como indicadores diretos de adequação, um modelo logit prevê apenas probabilidades que devem, então, ser comparadas com as escolhas reais. A medida $U^{2}$, usada para modelos probabilísticos, representa a fração de incerteza, ou o grau de variabilidade, empiricamente explicada pelo modelo relativo a uma distribuição previamente estabelecida de probabilidades de escolha. A distribuição prévia constitui o modelo nulo, que define a máxima entropia para a situação em exame. Um modelo que produz a mesma entropia que o modelo nulo possui um $U^{2}=0$. Um modelo que prevê corretamente $100 \%$ das observações apresenta um $U^{2}=1$, ou seja, prevê corretas probabilidades 0 e 1 . Assumindo-se que a distribuição prévia seja independente da ocasião de escolha, $U^{2}$ é dado por:

$U^{2}=1-\frac{\ln L_{\Omega}}{\ln L_{\omega}}$

onde $L_{\Omega}$ se refere ao máximo da função de verossimilhança, quando maximizada com relação a todos os parâmetros $\beta$ (incluindo o termo constante), e $L_{\omega}$ é o máximo da função de verossimilhança, quando maximizada em relação apenas ao termo constante (modelo nulo). 


\section{Resultados}

A Tabela 3 apresenta os parâmetros obtidos e seus respectivos desvios padrões. A análise de cada grupo de variáveis encontra-se a seguir.

Tabela 3: Parâmetros Estimados

\begin{tabular}{c|c|c|c}
\hline Parâmetro & Valor & Desvio Padrão & Estatística t \\
\hline$\beta_{01}$ & 0,62 & 2,52 & 0,25 \\
\hline$\beta_{02}$ & 0,18 & 3,77 & 0,05 \\
\hline$\beta_{04}$ & 2,70 & 2,45 & 1,10 \\
\hline$\beta_{1}$ & $-5,04$ & 4,83 & $-1,04$ \\
\hline$\beta_{2}$ & $-6,51$ & 6,28 & $-1,04$ \\
\hline$\beta_{3}$ & $-12,46$ & 6,48 & $-1,92^{\dagger}$ \\
\hline$\beta_{4}$ & $-16,35$ & 6,15 & $-2,66^{*}$ \\
\hline$\beta_{5}$ & 0,0007 & 0,0002 & $3,50^{*}$ \\
\hline$\beta_{6}$ & 0,0007 & 0,0003 & $2,33^{*}$ \\
\hline$\beta_{8}$ & $-0,0004$ & 0,0004 & $-1,00$ \\
\hline$\beta_{9}$ & 3,83 & 2,02 & $1,90^{\dagger}$ \\
\hline$\beta_{10}$ & 13,25 & 3,12 & $4,25^{*}$ \\
\hline$\beta_{11}$ & 12,44 & 4,37 & $2,85^{*}$ \\
\hline$\beta_{12}$ & 26,56 & 121,23 & 0,22 \\
\hline$\beta_{13}$ & 1,97 & 2,29 & 0,86 \\
\hline$\beta_{14}$ & 14,39 & 5,23 & $2,75^{*}$ \\
\hline$\beta_{15}$ & 5,39 & 2,80 & $1,93^{\dagger}$ \\
\hline$\beta_{16}$ & 21,94 & 102,54 & 0,21 \\
\hline
\end{tabular}

* significativo ao nível de confiança de 5\%; significativo ao nível de confiança de $10 \%$. $L_{\Omega}=-850,6 \cdot \mathrm{U}^{2}=0,626$.

Os coeficientes $\beta_{01}, \beta_{02}$ e $\beta_{04}$ são das constantes específicas às alternativas, ou seja, são os interceptos. Captam componentes específicos de cada marca, que não são captados por outras variáveis exógenas; e seriam zero se todas as variáveis que explicassem as diferenças entre as marcas fossem incluídas no modelo logit. O número de tais variáveis deve ser igual ao número de alternativas menos um. Escolheu-se nesta pesquisa, aleatoriamente, a marca $\mathrm{C}$ como a alternativa base, logo $\beta_{03}$ é zero para identificação do modelo. Três variáveis categóricas foram criadas com os valores de 1, 0, 0 para a marca A; 0, 1, 0 para B e 0, 0, 1 para D.

Preço: os coeficientes do preço, $\beta_{1}$ a $\beta_{4}$, apresentaram os sinais negativos 
esperados, indicando que o aumento do preço reduz a probabilidade de escolha das marcas. Mas somente para a marca D tal coeficiente foi significativo a 5\%. Esta é a marca de menor preço das quatro analisadas, provavelmente atingindo um segmento de clientes bastante sensível ao preço.

Renda: os coeficientes $\beta_{5}, \beta_{6}$ e $\beta_{8}$ referem-se à variável renda per capita para as marcas A, B e D, respectivamente. Esta é a única variável específica ao domicílio, e não à alternativa. Portanto, somente pode ser incorporada ao modelo logit condicional pelo uso dos termos de interação com variáveis categóricas. Caso contrário, o modelo não pode ser identificado, já que a variável possui valores idênticos para todas as alternativas.

Basicamente esta variável reflete as diferenças em preferências pelas marcas em função da renda per capita. Por falta de informação sobre alguma relação entre as marcas e a renda do domicílio, selecionou-se aleatoriamente a marca $\mathrm{C}$ como base, ou seja, o coeficiente $\beta_{7}$ é zero para identificação. A escolha da alternativa base é arbitrária, porque, caso outra alternativa seja definida como base, o sinal dos coeficientes é mudado, deixando-se inalteradas as diferenças nas utilidades e, conseqüentemente, as probabilidades de escolha.

O que interessa é o efeito da renda sobre a utilidade relativa de A, B e D. Como C é a marca mais barata, espera-se que as demais marcas recebam contribuições positivas de utilidade da variável renda em relação à marca C. Isto acontece nos coeficientes $\beta_{5}$ e $\beta_{6}$, que apresentaram significância ao nível de $5 \%$, indicando que o aumento de renda tem efeito significativo sobre a probabilidade de compra das marcas A e B, aquelas com maior preço e participação de mercado.

Lealdade: os coeficientes $\beta_{9}$ a $\beta_{12}$ referem-se à lealdade às marcas $\mathrm{A}, \mathrm{B}, \mathrm{C}$ e $\mathrm{D}$, respectivamente. Somente os coeficientes das marcas B e C mostraram-se significativos em nível de $5 \%$. O sinal positivo de $\beta_{10}$ e $\beta_{11}$ indica que a lealdade às marcas $\mathrm{B}$ e $\mathrm{C}$ influencia positivamente a probabilidade de suas escolhas.

Interação entre Preço e Lealdade: os coeficientes $\beta_{13}$ a $\beta_{16}$ representam esta interação para as marcas A, B, C e D, respectivamente. Somente $\beta_{14}$ e $\beta_{15}$ são estatisticamente significativos, em nível de $5 \%$ e $10 \%$, respectivamente. Isto indica que o coeficiente de inclinação do grupo de clientes leais à marca $\mathrm{B}$ será $\beta_{2+} \beta_{14}$ e o do grupo de leais à marca C será $\beta_{3+} \beta_{15}$, sendo $\beta_{2}$ e $\beta_{3}$ os coeficientes de inclinação do grupo dos não leais às marcas $\mathrm{B}$ e $\mathrm{C}$, respectivamente. Logo, para as marcas B e C os coeficientes de inclinação são 7,88 e -7,07, sinalizando que os clientes leais a estas marcas são menos sensíveis ao preço, em relação aos clientes não leais, que apresentaram coeficientes de -6,51 e -12,46, respectivamente. Por outro lado, não houve diferença significativa entre clientes leais e não leais para as marcas A e D. 
As Tabelas 4 e 5 resumem o ponto chave deste artigo, mostrando as elasticidadespreço da escolha (na diagonal) e a elasticidade-preço cruzada da escolha para clientes não leais e leais às marcas, respectivamente. Os sinais das elasticidades diretas (diagonal) são negativos, apontando que o aumento de preço da marca $p$ reduz a probabilidade de sua compra. Os sinais positivos das elasticidades cruzadas (fora da diagonal) implicam que um aumento de preço da marca $p$ causa um aumento da probabilidade de consumo das marcas substitutas na mesma categoria.

Tabela 4: Elasticidades-Preço da Escolha (na diagonal) e Cruzada da

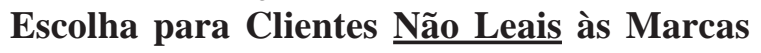

\begin{tabular}{lrrrr}
\hline & A & \multicolumn{1}{c}{ B } & \multicolumn{1}{c}{ C } & \multicolumn{1}{c}{ D } \\
\hline $\mathbf{A}$ & $\mathbf{- 0 , 4 0 8}$ & 1,777 & 1,777 & 1,777 \\
$\underline{\boldsymbol{B}}$ & 0,357 & $\mathbf{- 3 , 3 4 1}$ & 0,357 & 0,357 \\
$\underline{\boldsymbol{C}}$ & 0,191 & 0,191 & $\mathbf{- 2 , 5 9 9}$ & 0,191 \\
$\mathbf{D}$ & 0,098 & 0,098 & 0,098 & $\mathbf{- 5 , 2 6 1}$ \\
\hline
\end{tabular}

Tabela 5: Elasticidades-Preço da Escolha (na diagonal) e Cruzada da Escolha para Clientes Leais às Marcas

\begin{tabular}{rrrrr}
\hline & A & \multicolumn{1}{c}{ B } & \multicolumn{1}{c}{ C } & \multicolumn{1}{c}{ D } \\
\hline $\mathbf{A}$ & $\mathbf{- 0 , 4 0 8}$ & 1,777 & 1,777 & 1,777 \\
$\underline{\boldsymbol{B}}$ & 0,357 & $\mathbf{- 0 , 8 9 1}$ & 0,357 & 0,357 \\
$\underline{\boldsymbol{C}}$ & 0,191 & 0,191 & $\mathbf{- 0 , 9 9 8}$ & 0,191 \\
$\mathbf{D}$ & 0,098 & 0,098 & 0,098 & $\mathbf{- 5 , 2 6 1}$ \\
\hline
\end{tabular}

A hipótese de que os consumidores leais sejam menos sensíveis ao preço na escolha da marca do que os compradores não leais não foi rejeitada para duas marcas, B e C, que contam com participação de mercado e preços intermediários A segunda hipótese, de que a renda do consumidor influencia positivamente a probabilidade de escolha de marcas mais caras na categoria não foi rejeitada. Supondo-se preço e qualidade como altamente correlacionados, os consumidores buscam maior qualidade, dada a restrição orçamentária.

\section{Conclusão}

O foco deste artigo foi o efeito do preço na escolha da marca, levando-se em conta a lealdade do consumidor. Quanto ao objetivo principal, conclui-se que 
domicílios leais e não leais a uma marca de um bem de consumo geral podem ser diferentemente sensíveis às promoções de preços de suas marcas preferidas e das demais para a escolha da marca. Tal sensibilidade varia de marca para marca.

A implicação gerencial disto é que gerentes de categoria de redes varejistas, com a posse de dados escaneados, podem tomar decisões específicas de promoção de preços para cada marca. Por exemplo, se para uma determinada marca clientes leais não são sensíveis à variação de preço para a escolha da marca, promoções de preços que visem à troca de marca pelo cliente (típico em caso de marcas próprias dos supermercados) podem ser ineficazes. Caso a empresa segmente seus clientes por níveis de lealdade e as marcas analisadas sejam do tipo A e D com relação a EPEM, estratégias de promoção de preços podem não ser inteligentes, se aplicadas a segmentos diferentes, quando visam à mudança de marca.

Na questão da renda domiciliar e preços das marcas escolhidas pelos domicílios, os resultados indicam que domicílios com rendas mais altas apresentam maior probabilidade de escolha de marcas de preço mais alto, como esperado. Partindose do pressuposto de que há alta correlação entre preço e qualidade (ALLEMBY; ROSSI, 1991), os domicílios estudados estão maximizando sua utilidade também pela busca de qualidade, de acordo com a tradicional literatura mercadológica (BEN-AKIVA; LERMAN, 1985, p. 361).

Há limitações inerentes à natureza da pesquisa realizada, principalmente deficiências metodológicas e da característica dos dados. Primeiramente, todo o estudo empírico, baseado em dados escaneados, apesar de terem inúmeras vantagens, não fornecem informações psicográficas e atitudinais do comportamento de compra. Um viés percebido foi o não controle das compras feitas pelo domicílio em locais fora da rede supermercadista analisada. Finalmente, algumas lojas podem apresentar promoções relâmpago em determinados dias (geralmente sábados), que duram apenas alguns minutos e podem ser anunciadas na loja. Tais promoções não foram capturadas nesta pesquisa. Este fato também pode ser fonte de viés.

Entretanto este artigo oferece subsídios para o conhecimento do comportamento de compra de domicílios quando eles escolhem uma marca numa categoria, diante do uso de promoções freqüentes de preço, possibilitando decisões mais inteligentes por parte dos profissionais de mercado. Ademais, a base metodológica apresentada aqui, com o uso de dados escaneados, pode auxiliar profissionais de marketing a estimarem a elasticidade-preço da escolha da marca, estimativa pouco usual no varejo brasileiro. 
Por tratar-se de pesquisa empírica, o desenvolvimento teórico foi necessário para delinear condições em que outras hipóteses pudessem ser derivadas. Sugestões para pesquisas futuras incluem a modelagem de outras decisões de compra, que são influenciadas pela sensibilidade ao preço, como a quantidade comprada, a época e local de compra, incluindo decisões de quanto, quando e onde comprar.

\section{Artigo recebido em 09.01.2004. Aprovado em 21.06.2004.}

\section{NotA}

${ }^{1}$ Os autores são gratos à Coordenação de Aperfeiçoamento de Pessoal de Nível Superior (CAPES) pela bolsa concedida ao primeiro autor para a realização deste estudo.

\section{RefERÊnCIAS Bibliográficas}

AINSLIE, A.;

ROSSI, P.E.

Similarities in choice behavior across product categories. Marketing Science, Linthicum, v. 17, n. 2, p. 91106, Feb. 1998.

\section{ALLEMBY, G. M.;}

\section{ROSSI, P. E.}

Quality perceptions and asymmetric switching between brands. Marketing Science, Linthicum, v. 10, n. 3, p. 185204, summer 1991.

\section{ARBEX, G. G.}

Automação dispara nos supermercados. Gazeta Mercantil, São Paulo, ano LXXX - n. 21-951, p. 4-17, out. 2000.

ASSOCIAÇÃO BRASILEIRA DE SUPERMERCADOS.

Ranking ABRAS 2002, São Paulo, 2002. Disponível em: <http:// www.abrasnet.com.br>. Acesso em: 8 dez. 2002.
BELL, D.;

LATTIN, J. M.

Shopping behavior and consumer preference for for store price format: why large basket shoppers prefer EDLP. Marketing Science, Linthicum, v. 17, n. 1, p. 66-88, 1998.

BEN-AKIVA, M.;

LERMAN, S. R.

Discrete choice analysis. 1st ed. Cambridge: MIT Press, 1985. p. 31-87.

BLATTBERG, R. et al. Identifying the deal prone segment. Journal of Marketing Research, Chicago, v. 15, n. 3, p. 369-377, Aug. 1978.

BUCKLIN, R.;

GUPTA, $\mathrm{S}$.

Brand choice, purchase incidence, and segmentation: an integrated approach. Journal of Marketing Research, Chicago, v. 29, n. 2, p. 201215, May 1992. 
CHIANG, J.

A simultaneous approach to the whether, what and how much to buy questions. Marketing Science, Linthicum, v. 10, n. 4, p. 297-315, fall 1991.

\section{CHINTAGUNTA, P. K.;}

KYRIAZIDOU, E.;

PERTOLD, J.

Panel data analysis of household brand choices. Journal of Econometrics, Amsterdam, v. 103, n. 1-2, p. 111-153, July 2001.

FUNDAÇÃO INSTITUTO DE PESQUISAS ECONÔMICAS.

Índices. São Paulo. Disponível em: $<$ http://www.fipe.com.br>. Acesso em: 9 jan. 2004.

GUADAGNI, P. M.;

LITTLE, J. D. C.

A logit model of brand choice calibrated on scanner data. Marketing Science, Linthicum, v. 2, n. 3, p. 203-38, summer 1983.

GUJARATI, D.

Econometria básica. São Paulo: Makron Books, 2000. p. 516-518.

\section{GUPTA, S.}

Impact of sales promotion on when, what, and how much to buy. Journal of Marketing Research, Chicago, v. 25, n. 4, p. 342-55, Nov. 1988.
JOHNSTON, J.;

DINARDO, J.

Econometric methods. 4 th ed. New York: McGraw-Hill, 1997. p. 388-391.

KRISHNAMURTHI, L.;

RAJ, S. P.

A model of brand choice and purchase quantity price sensitivities. Marketing Science, Linthicum, v. 7, n. 1, p. 1-20, winter 1988.

An empirical analysis on the relationship between brand loyalty and consumer price elasticity. Marketing Science, Linthicum, v. 10, n. 2, p. 172-183, spring 1991.

LIPSEY, R. G;

COURANT, P. N.;

RAGAN, C. T. S.

Economics. 12th ed. London: Addisson-Wesley, 1999. p. 332.

ROCHA, A.;

CHRISTENSEN, C.

Marketing: teoria e prática no Brasil.

2. ed. São Paulo: Atlas, 1999. p. 108.

SIMON, H.

Price management. 2nd ed. Amsterdam: Elsevier Science Publishers, 1989. p. 8-40.

\section{TELLIS, G.}

Advertising exposure, loyalty, and brand purchase: a two stage model of choice. Journal of Marketing Research, Chicago, v. 25, n. 2, p. 134144, May 1988. 